\title{
Acute Effects of Triathlon Race on Oxidative Stress Biomarkers
}

\author{
Simona Mrakic-Sposta $\mathbb{D}^{\mathbb{D}},{ }^{1}$ Maristella Gussoni $\mathbb{D},{ }^{2}$ Alessandra Vezzoli, ${ }^{1}$ Cinzia Dellanoce, ${ }^{1}$ \\ Mario Comassi, ${ }^{3}$ Guido Giardini, ${ }^{4}$ Rosa Maria Bruno $\mathbb{D}^{5},{ }^{5}$ Michela Montorsi $\left(\mathbb{D},{ }^{6}\right.$ \\ Anca Corciu, ${ }^{3}$ Fulvia Greco, ${ }^{2}$ and Lorenza Pratali ${ }^{3}{ }^{3}$ \\ ${ }^{1}$ Institute of Clinical Physiology, National Council of Research (IFC-CNR), ASST Grande Ospedale Metropolitano Niguarda, \\ Milan, Italy \\ ${ }^{2}$ Institute of Science and Chemical Technology, National Council of Research (SCITEC-CNR), Milan, Italy \\ ${ }^{3}$ Institute of Clinical Physiology, National Council of Research (IFC-CNR), Pisa, Italy \\ ${ }^{4}$ Neurology and Neurophysiology Department, Mountain Medicine Center Valle d' Aosta Regional Hospital Umberto Parini, \\ Aosta, Italy \\ ${ }^{5}$ Department of Clinical and Experimental Medicine, University of Pisa, Italy \\ ${ }^{6}$ Department of Human Sciences and Promotion of the Quality of Life, San Raffaele Roma Open University, Milan, Italy
}

Correspondence should be addressed to Lorenza Pratali; lorenza@ifc.cnr.it

Received 11 September 2019; Accepted 12 November 2019; Published 17 January 2020

Academic Editor: Luigi Iuliano

Copyright (c) 2020 Simona Mrakic-Sposta et al. This is an open access article distributed under the Creative Commons Attribution License, which permits unrestricted use, distribution, and reproduction in any medium, provided the original work is properly cited.

\begin{abstract}
The response to strenuous exercise was investigated by reactive oxygen species (ROS) production, oxidative damage, thiol redox status, and inflammation assessments in 32 enrolled triathlon athletes $\left(41.9 \pm 7.9\right.$ yrs) during Ironman ${ }^{\circledR}$ (IR), or half Ironman ${ }^{\circledR}$ (HIR) competition. In biological samples, inflammatory cytokines, aminothiols (glutathione (GSH), homocysteine (Hcy), cysteine (Cys), and cysteinylglycine (CysGly)), creatinine and neopterin, oxidative stress (OxS) biomarkers (protein carbonyl (PC), thiobarbituric acid-reactive substances (TBARS)), and ROS were assessed. Thirteen HIR and fourteen IR athletes finished the race. Postrace, ROS (HIR +20\%; IR +28\%; $p<0.0001$ ), TBARS (HIR $+57 \%$; IR $+101 \%)$, PC (HIR $+101 \%$; IR $+130 \%)$ and urinary neopterin (HIR $+19 \%$, IR $+27 \%$ ) significantly (range $p<0.05-0.0001$ ) increased. Moreover, HIR showed an increase in total Cys $+28 \%$, while IR showed total aminothiols, Cys, Hcy, CysGly, and GSH increase by $+48,+30,+58$, and $+158 \%$, respectively (range $p<0.05-0.0001)$. ROS production was significantly correlated with TBARS and PC $\left(R^{2}=0.38\right.$ and $R^{2}=0.40$; $p<0.0001$ ) and aminothiols levels (range $R^{2}=0.17-0.47$; range $p<0.01-0.0001$ ). In particular, ROS was directly correlated with the athletes' age $\left(R^{2}=0.19 ; p<0.05\right)$, with ultraendurance years of training $\left(R^{2}=0.18 ; p<0.05\right)$ and the days/week training activity $\left(R^{2}=0.16 ; p<0.05\right)$. Finally, the days/week training activity (hours/in the last 2 weeks) was found inversely correlated with the IL-6 postrace $\left(R^{2}=-0.21 ; p<0.01\right)$. A strenuous performance, the Ironman ${ }^{\circledR}$ distance triathlon competition, alters the oxidant/antioxidant balance through a great OxS response that is directly correlated to the inflammatory parameters; furthermore, the obtained data suggest that an appropriate training time has to be selected in order to achieve the lowest ROS production and IL-6 concentration at the same time.
\end{abstract}

\section{Introduction}

Triathlon is a multiple-stage competition involving the completion of three continuous and sequential endurance disciplines [1]. Even if many variations of the sport exist, triathlon, in its most popular form, involves swimming, cycling, and running in immediate succession over various distances [2]. Triathlon thereby provides a great opportunity to investigate the effects of multidisciplinary exercises on health, and to this aim, much remains to be done [3]. Long competitions require great power endurance possibly leading to heat stress and dehydration $[4,5]$, muscle injury [6], oxidative stress 
$[7,8]$, inflammation $[9,10]$, immunologic alterations [11, 12], and cardiac remodeling [13].

Aerobic exercises, particularly ultraendurance exercise, increase the oxygen consumption rate, which, in turn, increases the high reactive oxygen species (ROS) production $[14,15]$ so antioxidant defenses are mostly required to protect cells from oxidative damage. Overproduction of ROS, potentially damaging cells, particularly macromolecules (i.e. lipids, proteins, carbohydrates, and nucleic acids) $[16,17]$ in both skeletal muscle and blood [18], is known as oxidative stress (OxS). As widely reported, OxS may result in an inflammatory response by the immune system to protect tissues $[12,19,20]$. Nonetheless, at appropriate concentration, ROS are known to act as important signaling molecules [21, 22].

ROS measurement is difficult because they are produced in low quantities, characterized by extremely short half-life (i.e., $\mathrm{t}_{1 / 2}$ : superoxide $\left[\mathrm{O}_{2}{ }^{-}\right]=10^{-4} \mathrm{~s}$; nitric oxide $\left[\mathrm{NO}^{-}\right]=0.4 \mathrm{~s}$ at ambient temperature) and rapid interaction with antioxidants, like thiol residues, and cellular components, molecular oxygen, and metalloproteins. Numerous assays and biomarkers have been used to measure oxidative stress $[16,17]$. However, none of them is entirely satisfactory and/or they have been inappropriately used [23].

Electron paramagnetic resonance (EPR) is the only technique capable of providing a direct detection of the "instantaneous" presence of free radical species in a sample $[21,24]$ and at the same time giving an "intrinsically quantitative" information by adopting a microinvasive measuring method [25]. Indeed, as proportional to the number of the excited electron spins, EPR signal areas can return absolute concentrations once that a stable radical compound is adopted as an internal reference.

Aminothiols are a major species of nonenzymatic antioxidant compounds characterized by reduced sulfhydryl moieties able to directly quench ROS. The specimens are present in all fluids and tissues, furthermore playing important roles in cell signaling, metabolism, and detoxification, and their concentration is associated to OxS [26]. Various aminothiols (i.e., homocysteine (Hcy), cysteine (Cys), cysteinylglycine (CysGly)) are metabolically strictly connected and can be considered the principal interface between changing redox environment and protein activity [8]. Reduced glutathione (GSH) results in the most abundant presence of aminothiols in tissues, and it serves multiple functions to protect from oxidative damage by keeping the intracellular environment in a reduced state [27].

Several studies have investigated alterations of the biochemical responses in athletes involved in ultraendurance exercise, focusing the interest on exercise-induced muscle damage accompanied by the presence of inflammatory mediators and how their modulation can be affected by the intensity, mode, and duration of the exercise challenge [28-30].

Some of these have in particular determined the cytokine responses to ultraendurance exercise, where the immune response can be affected by ROS [30] that are, in turn, able to modulate the acute phase of inflammatory responses as well [31].
Another marker providing information about immune activation is urinary neopterin $[32,33]$. Neopterin is an unconjugated pteridine, secreted in large quantities by activated macrophages: it can be used as a clinical marker of activated cellular immunity [33].

In order to clarify the physiological mechanisms involved when the human body is pushed acutely to its limits, a group of athletes performing in the Ironman ${ }^{\circledR}$ triathlon distance competition (IR) and half distance Ironman ${ }^{\circledR}$ triathlon (HIR) were monitored in the present study. In particular, as redox physiology plays an important role in the performance, especially under endurance exercise, redox status modifications in the time course of the competition were investigated by measuring ROS production levels, lipids, and protein OxS damage biomarkers (TBARS and PC), and thiols' redox status in both erythrocytes and plasma. Finally, to complete the picture, inflammatory markers (IL-6, IL-10, IL $1 \beta$, TNF- $\alpha$, Irisin, and Monocyte Chemoattractant Protein-1), biochemical-hematological profile, and renal function markers (creatinine and neopterin) were assessed. Anthropometrical and training load characteristics influences were analyzed too.

\section{Materials and Methods}

2.1. Snapshot of the Race. The study was performed during the "Elbaman ${ }^{\circledR}$ " race at Elba Island (Italy). Two types of competition were proposed by the organizers (http://www .elbaman.it): "Elbaman" (IR): $3.8 \mathrm{~km}$ swim, followed by $180 \mathrm{~km}$ bike and $42.2 \mathrm{~km}$ run, and at the same time, a competition on half the distance, the Elbaman73 (HIR): $1.9 \mathrm{~km}$ swim, followed by $94 \mathrm{~km}$ bike and $21.1 \mathrm{~km}$ run. A sketch of the experimental protocol and of the race is displayed in Figure 1. On the day of the race, ambient temperature was $23.2^{\circ} \mathrm{C}$. During the competition, athletes were allowed to eat and to drink ad libitum what was provided by the organizers.

2.2. Subjects. Thirteen HIR (age $42.9 \pm 8.8$ yrs; height 174.3 $\pm 5.6 \mathrm{~cm}$ ) and nineteen IR (age $41.3 \pm 7.1$ yrs; height 174.9 $\pm 4.6 \mathrm{~cm}$ ) athletes were recruited on a volunteer basis among male participants in the competitions. The exclusion criteria were as follows: age $<18$ yrs; presence of chronic disease such as hypertension, hypercholesterolemia, and diabetes; use of any medication, antioxidant, or related supplements in the 7 days preceding the study; and unwillingness to sign informed consent. The anthropometric parameters, body mass index (BMI), body fat (FM), free fat masses (FFM), and total body water (TBW) were determined by bipolar bio-impedentiometry (TBF-300A Body Composition Analyzer; Tanita Corporation, Arlington Heights, IL, USA). Before and immediately after the race, blood pressure (BP) was measured by a standard cuff sphygmomanometer, finger $\mathrm{O}_{2}$ saturation $\left(\mathrm{SaO}_{2}\right)$ and heart rate (HR) ( $\mathrm{HR}$ and $\mathrm{SaO}_{2}$ : by Oximetry-Ohmeda TuffSat, GE Healthcare, Helsinki, Finland) were measured, and the mean value of three evaluations for each parameter was obtained.

The triathletes enrolled were asked to not perform any form of exercise $24 \mathrm{~h}$ preceding the race. 


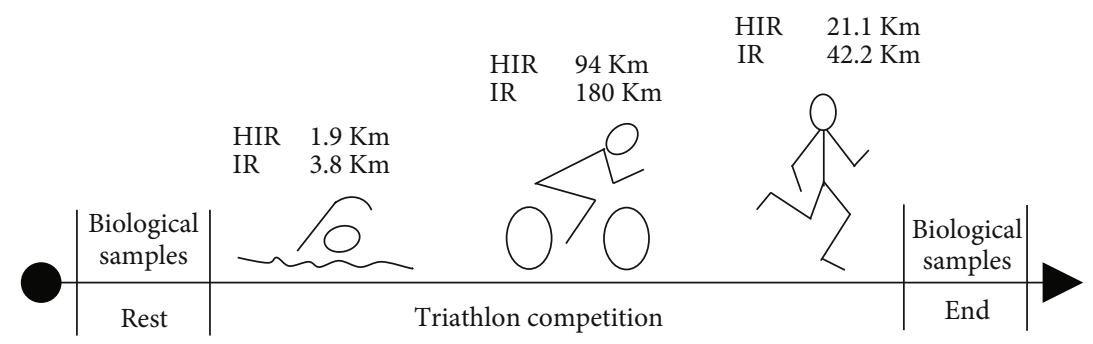

FIgURE 1: Experimental protocol adopted to measure the OxS parameters on the biological samples collected from the Elbaman (IR) and Elbaman 73 (HIR) selected participants.

Each of the enrolled athletes, with training history and training load for the last two weeks before the race, was administered.

The experimental protocol was approved by the Valle d'Aosta Region Ethical Committee (n. 65943), and all volunteers signed an informed consent according to the Declaration of Helsinki.

2.3. Blood and Urine Sample Collection. Athletes underwent two test sessions for venous, capillary blood, and urine sample collections: the first (PRE) was performed 1 day before, while the second (POST) immediately after the end of the race. Approximately $15 \mathrm{~mL}$ of blood was drawn from an antecubital vein and collected in heparinized $(5 \mathrm{~mL})$ and EDTA- $(10 \mathrm{~mL})$ treated vacutainer tubes (Becton Dickinson and Company, UK). Plasma was separated by centrifugation (5702R, Eppendorf, Germany) at $3000 \mathrm{~g}$ for $5 \mathrm{~min}$ at $4^{\circ} \mathrm{C}$. Plasma and erythrocytes samples were immediately stored in multiple aliquots at $-80^{\circ} \mathrm{C}$ until being assayed. Urine samples were also collected both PRE and POST, and aliquots were stored at $-80^{\circ} \mathrm{C}$ until analyses. Samples were thawed only once for analyses, which were performed within two weeks from collection. $50 \mu \mathrm{L}$ of capillary blood was taken from the fingertip PRE and POST for EPR measurements.

\subsubsection{Blood Measurements}

(1) Hematological Parameters. Hematological parameters (i.e., cholesterol, osmolality, uric acid, and main types of the white blood cells (WBC)) were analyzed by standard methods; inflammatory biomarkers (i.e., TNF- $\alpha$, IL- $1 \beta$, IL6, IL-10, Monocyte Chemoattractant Protein-1 (MCP-1), and Irisin) were assessed by enzyme-linked immune assays according to the manufacturer's instructions. Detailed procedures and the assessed values for each parameter above reported have been previously reported by some of us [34].

(2) Oxidative stress $(O x S)$.

(i) ROS Detection. ROS production rate was assessed using the methods of Mrakic-Sposta et al. [7, 25, 35 , 67] with an X-band EPR instrument (E-ScanBruker BioSpin, GmbH, MA USA), using $50 \mu \mathrm{L}$ of capillary blood withdrawn from the fingertip and treated immediately with probe solution CMH (1-hydroxy-3-methoxycarbonyl-2,2,5,5-tetra- methylpyrrolidine). All samples were stabilized at $37^{\circ} \mathrm{C}$ by the Temperature and Gas Controller "Bio III" unit, interfaced to the spectrometer. All spectra were collected by acquisition parameters previously reported [7, 25, 35, 67], and Bruker software (Win EPR System, V. 2.11) was adopted for the analysis.

(ii) Thiols Assessment. Total and reduced aminothiols were measured in the erythrocytes and plasma samples according to previously validated methods [36]. Briefly, Tris-(2-carboxyethyl)-phosphine hydrochloride (TCEP) and 4-fluoro-7-sulfamoylbenzofurazan (ABD-F) were used as reducing and derivatizing agents, respectively; reduced aminothiols were assessed by mixing erythrocytes and plasma with $10 \%$ trichloroacetic acid $(1: 1(v / v))$. $100 \mu \mathrm{L}$ of each supernatant obtained was added with $10 \mu \mathrm{L}$ of $0.4 \mathrm{M} \mathrm{NaOH}, 70 \mu \mathrm{L}$ of $1 \mathrm{M}$ borate buffer $\mathrm{pH} 11$ containing $4 \mathrm{mM}$ EDTA, $30 \mu \mathrm{L}$ of $1 \mathrm{M}$ borate buffer pH 9.5 containing $4 \mathrm{mM}$ EDTA, and $10 \mu \mathrm{L}$ of $10 \mathrm{~g} \cdot \mathrm{L}^{-1} \mathrm{ABD}-\mathrm{F}$ in borate buffer $(1 \mathrm{M}$, pH 9.5 containing $4 \mathrm{mM}$ EDTA). Samples were incubated at $4^{\circ} \mathrm{C}$ for $90 \mathrm{~min}$, and then $10 \mu \mathrm{L}$ was injected into the High-Performance Liquid Chromatography (HPLC) system for analysis. Thiols' separation was performed at room temperature by isocratic HPLC analysis on a Discovery C-18 column $(250 \times 4.6 \mathrm{~mm} \mathrm{ID}$, Supelco, Sigma-Aldrich $)$, eluted with a solution of $0.1 \mathrm{~mol} \cdot \mathrm{L}^{-1}$ acetate buffer, pH 4.0: methanol, $81: 19(v / v)$, at a flow rate of $1 \mathrm{~mL} \cdot \mathrm{min}^{-1}$. Fluorescence intensities were measured by using a fluorescence spectrophotometer (JASCO, Japan, excitation at $390 \mathrm{~nm}$ and emission at $510 \mathrm{~nm}$ ). A standard calibration curve was used to assay sample concentration. The concentration of the oxidized forms was calculated as the difference between the total and the reduced forms

(iii) Protein Carbonyls (PC). The accumulation of oxidized proteins was measured by the reactive carbonyl content. A protein carbonyl (PC) assay kit (Cayman Chemical, U.S.) was used to colorimetrically evaluate the oxidized proteins at $370 \mathrm{~nm}$. The obtained values were normalized to the total protein concentration in a final pellet (absorbance reading at $280 \mathrm{~nm}$ ), in order to consider the protein loss during the washing steps, as suggested by the kit's user manual 
(iv) Thiobarbituric Acid-Reactive Substances (TBARS). Thiobarbituric acid-reactive substance (TBARS) measurement was adopted to detect lipid peroxidation. The used TBARS assay kit (Cayman Chemical, U.S.) allows a rapid photometric thiobarbituric acid malondialdehyde (TBAMDA) adduct detection at $532 \mathrm{~nm}$. A linear calibration curve was computed from pure malondialdehyde containing solutions

2.3.2. Urine Marker Measurements. As the collection of the $24 \mathrm{~h}$ urine is not possible, urinary parameters are standardized basing on the amount of the excreted creatinine, since, as well known, in a human subject and in the absence of renal disease, creatinine excretion rate keeps relatively constant.

(3) Creatinine and Neopterin. Creatinine and neopterin concentrations were measured by an isocratic high-pressure liquid chromatography (HPLC) method. Briefly, urine samples were thawed and centrifuged at $13000 \mathrm{rpm}$ for $5 \mathrm{~min}$ at $4^{\circ} \mathrm{C}$; the supernatant was then adequately diluted with the chromatographic mobile phase $\left(15 \mathrm{mM}\right.$ of $\left.\mathrm{K}_{2} \mathrm{HPO}_{4}, \mathrm{pH} 3.0\right)$. Neopterin and creatinine levels were measured using a Varian pump (240, autosampler ProStar 410) coupled to a fluorometric detector (JASCO FP-1520, $\lambda_{\mathrm{ex}}=355 \mathrm{~nm}$ and $\lambda_{\mathrm{em}}=450 \mathrm{~nm}$ ) for neopterin and to a UV-VIS detector (Shimadzu SPD 10-AV, $\lambda=240 \mathrm{~nm}$ ) for creatinine determinations. Neopterin and creatinine separations were performed at $50^{\circ} \mathrm{C}$ on a $5 \mu \mathrm{m}$ Discovery $\mathrm{C} 18$ analytical column $(250 \times 4.6 \mathrm{~mm}$ ID, Supelco, Sigma-Aldrich $)$ at a flow rate of $0.9 \mathrm{~mL} \cdot \mathrm{min}^{-1}$. Linear calibration curves were found over a concentration of $0.125^{-1} \mathrm{mmol} \cdot \mathrm{L}^{-1}$ and $1.25^{-10} \mathrm{mmol} \cdot \mathrm{L}^{-1}$ for neopterin and creatinine (the score in $\mu \mathrm{mol} \cdot \mathrm{L}^{-1}$ was divided by 88.4 to obtain $\mathrm{mg} \cdot \mathrm{dL}^{-1}$ creatinine), respectively. Interassay and intra-assay variation coefficients resulted in less than the $5 \%$.

2.4. Statistical Analysis. Data were reported as the mean $\pm \mathrm{SD}$ . Statistical analysis was performed by using the GraphPad Prism package (GraphPad Prism 8, Software Inc., San Diego, CA). The normality of the data distribution was tested with Shapiro-Wilk's test. The data were normally distributed. Experimental data were compared using ANOVA repeated measures with a Bonferroni posthoc test. Pearson's product moment correlation coefficient $(R)$ with $90 \%$ confidence intervals (CI) was used to determine the relationships between selected parameters. Prospective power calculation to determine the significant subject number was made by using the Freeware $\mathrm{G} *$ Power software (http://www.psycho .uni-duesseldorf.de/abteilungen/aap/gpower3/). At a power of $80 \%$, a significant population of 11 subjects was calculated, well below the subject's population recruited for the study.

\section{Results}

3.1. Anthropometric, Physiological, and BiochemicalHematological Parameters. Among 32 enrolled athletes, 27 completed the competition. Anthropometric and physiological parameters are reported in Table 1. At POST, weight, FFM, and TBW were found to be decreased in both the IR and HIR groups (of about the $3 \%$ ). As expected, heart rate was significantly increased immediately after the end of the race: IR: $+37 \%$ and HIR: $+41 \%$.

White blood cell (WBC) count and the percentages of the five types of WBCs in overall count recorded at PRE and POST race are shown in Table 2. Interleukins (IL-1 $\beta$; IL-6, IL-10, and TNF- $\alpha$ ) and cytokine (MCP-1) levels were also reported.

3.2. ROS Production, Oxidative Damage, and Aminothiol Measurements. ROS production rate measured in capillary blood and plasmatic OxS biomarkers concentration data is shown in the plot panels of Figure 2. No significant differences in the ROS production rate and oxidative damage biomarkers (TBARS and PC) at basal levels were found between the two (HIR and IR) groups.

The ROS production significantly increased at POST vs. PRE $(\mathrm{p}<0.0001)$ in HIR $(1.93 \pm 0.21$ vs. $1.62 \pm 0.10 \mu \mathrm{mol} \cdot-$ $\left.\min ^{-1}\right)$ and IR $\left(2.15 \pm 0.16\right.$ vs. $\left.1.68 \pm 0.11 \mu \mathrm{mol} \cdot \mathrm{min}^{-1}\right)$ athletes (Figure 2(a)), with significant differences in POST between HIR and IR.

A similar trend POST vs. PRE observation for oxidative damage biomarkers was observed for TBARS in HIR: $14.09 \pm 2.70$ vs. $8.91 \pm 1.72 \mu \mathrm{M}$, and in IR: $16.31 \pm 2.90$ vs. $8.09 \pm 2.80 \mu \mathrm{M}(p<0.0001)$ (Figure 2(b)); PC in HIR: 1.39 \pm 0.41 vs. $0.69 \pm 0.21$, and in IR: $1.54 \pm 0.51$ vs. $0.68 \pm$ $0.21 \mathrm{nmol} \cdot \mathrm{mg}^{-1}$ protein $(p<0.0001)$ (Figure 2(c)).

Aminothiol concentration data measured at PRE and POST sessions from HIR and IR subjects on both plasma and erythrocytes samples are reported in Table 3. At PRE, no significant differences were observed between the two athletes' groups. In plasma, significant $(p<0.001)$ changes at POST vs. PRE, in both the HIR and IR in total and oxidized Cys, were calculated. Furthermore, in IR at POST total Hcy $(p<0.05)$, total and oxidized CysGly $(p<0.001)$, and total and oxidized GSH $(p<0.0001)$, concentrations were found to be significantly increased.

In red blood cells (RBCs), total and reduced GSH concentration data resulted in a significant $(p<0.0001)$ increase in POST vs. PRE, in both the athletes' groups.

3.3. Neopterin. The renal functional biomarker neopterin data collected on both the HIR and IR athletes' groups at PRE and POST measurement sessions are shown in Figure 3. No significant differences were found at PRE in the two groups. Neopterin concentration significantly increased (POST vs. PRE) in both HIR: $86.88 \pm 8.78$ vs. $73.01 \pm 10.21(p<0.05)$, and IR $108.65 \pm 20.56$ vs. $85.87 \pm$ $11.89(p<0.001) \mu \mathrm{mol} \cdot \mathrm{mol}^{-1}$ creatinine. A significant difference was found for neopterin POST HIR and IR as well $(p<0.01)$.

3.4. Parameter Correlations. In both the HIR and IR groups, the correlations between OxS biomarker data (i.e., ROS production, TBARS, and PC concentrations) and biochemicalhematological parameters were observed. The calculated correlation coefficients $\left(R^{2}\right)$ and the significance levels are reported in Table 4. 
TABLE 1: Anthropometric and physiological parameters from the HIR and IR subjects who ended the race collected at PRE and POST measurement sessions.

\begin{tabular}{|c|c|c|c|c|c|c|}
\hline & PRE & $\begin{array}{c}\text { HIR } \\
(n=13) \\
\text { POST }\end{array}$ & $p$ value & PRE & $\begin{array}{c}\text { IR } \\
(n=14) \\
\text { POST }\end{array}$ & $p$ value \\
\hline Weight (kg) & $71.2 \pm 7.4$ & $69.5 \pm 7.7$ & $<0.0001$ & $70.2 \pm 5.5$ & $68.2 \pm 5.1$ & 0.0002 \\
\hline BMI $\left(\mathrm{kg} \cdot \mathrm{m}^{-2}\right)$ & $23.4 \pm 1.9$ & $22.8 \pm 2.0$ & $<0.0001$ & $22.9 \pm 1.4$ & $22.3 \pm 1.3$ & ns \\
\hline FM (kg) & $5.3 \pm 2.7$ & $5.5 \pm 2.4$ & ns & $5.1 \pm 2.5$ & $5.5 \pm 2.0$ & ns \\
\hline FFM (kg) & $66.1 \pm 5.9$ & $64.1 \pm 6.0$ & $<0.0001$ & $65.1 \pm 5.6$ & $62.7 \pm 5.8$ & $<0.0001$ \\
\hline TBW (\%) & $48.2 \pm 4.3$ & $47.0 \pm 4.4$ & $<0.0001$ & $47.6 \pm 4.1$ & $45.9 \pm 3.5$ & $<0.0001$ \\
\hline HR (bpm) & $60.5 \pm 8.7$ & $85.2 \pm 17.2$ & 0.0002 & $59.1 \pm 17.4$ & $80.8 \pm 14.2$ & 0.0039 \\
\hline SBP (mmHg) & $125.5 \pm 23.4$ & $141.2 \pm 11.5$ & ns & $123.7 \pm 16.9$ & $138.4 \pm 12.3$ & $\mathrm{~ns}$ \\
\hline DBP $(\mathrm{mmHg})$ & $72.5 \pm 13.5$ & $81.2 \pm 6.4$ & ns & $72.5 \pm 10.7$ & $79.5 \pm 16.7$ & ns \\
\hline $\mathrm{SaO} 2(\%)$ & $97.8 \pm 0.6$ & $96.6 \pm 1.3$ & ns & $97.6 \pm 0.9$ & $97.4 \pm 1.5$ & $\mathrm{~ns}$ \\
\hline
\end{tabular}

BMI: body mass index; FM: fat mass; FFM: fat-free mass; TBW: total body water; HR: heart rate; SBP: systolic blood pressure; DBP: diastolic blood pressure; $\mathrm{SaO}_{2}$ : arterial oxygen saturation (\%). Data are reported as the mean \pm SD. ns: not significant level.

TABLE 2: White blood cell (WBC) count $\left(10^{3} / \mu \mathrm{L}\right)$ and inflammatory markers: IL- $1 \beta$, IL-6, IL-10, TNF- $\alpha$, and MCP- 1 (pg/mL), determined at PRE and POST sessions from HIR and IR athletes. Data are reported as the mean \pm SD. The types of leukocytes, classified in the standard ways, are reported as a percentage (\%) of the total WBC together with the significance levels (POST vs. PRE).

\begin{tabular}{|c|c|c|c|c|c|c|}
\hline & \multicolumn{3}{|c|}{ HIR } & \multicolumn{2}{|c|}{ IR } & \multirow[b]{2}{*}{$p$ value } \\
\hline & PRE & POST & $p$ value & PRE & POST & \\
\hline $\mathrm{WBC}\left(10^{3} / \mu \mathrm{L}\right)$ & $6.45 \pm 3.29$ & $14.27 \pm 3.19$ & $<0.05$ & $5.23 \pm 0.83$ & $16.85 \pm 2.37$ & $<0.05$ \\
\hline \multicolumn{7}{|c|}{ Leukocytes (\%) } \\
\hline Neutrophils & 60 & 77 & $<0.0001$ & 58 & 78 & $<0.0001$ \\
\hline Eosinophils & 2.3 & 3 & ns & 2.2 & 2.7 & ns \\
\hline Basophiles & 0.4 & 0.5 & ns & 0.4 & 0.5 & ns \\
\hline Lymphocytes & 31 & 11 & $<0.05$ & 33.5 & 11.4 & $<0.05$ \\
\hline Monocytes & 6.6 & 8.5 & 0.0013 & 5.8 & 7 & $<0.0001$ \\
\hline \multicolumn{7}{|c|}{${ }^{*}$ Inflammatory markers $(\mathrm{pg} / \mathrm{mL})$} \\
\hline IL- $1 \beta$ & $17.7 \pm 16.5$ & $30.2 \pm 47.0$ & $<0.05$ & $20.9 \pm 17.1$ & $25.3 \pm 21.7$ & ns \\
\hline IL-6 & $8.9 \pm 18.8$ & $18.9 \pm 13.7$ & $<0.05$ & $4.2 \pm 2.7$ & $18.9 \pm 13.1$ & $<0.05$ \\
\hline IL-10 & $14.2 \pm 19.2$ & $35.0 \pm 32.8$ & $<0.05$ & $11.5 \pm 6.6$ & $63.5 \pm 65.9$ & $<0.05$ \\
\hline TNF- $\alpha$ & $19.0 \pm 30.0$ & $19.0 \pm 18.9$ & ns & $10.9 \pm 4.3$ & $12.7 \pm 3.1$ & ns \\
\hline MCP-1 & $312 \pm 139$ & $852 \pm 284$ & $<0.05$ & $337 \pm 165$ & $1116 \pm 598$ & $<0.05$ \\
\hline
\end{tabular}

${ }^{*}$ Inflammatory marker data were previously reported by some of us [34].

The linear relationships of the OxS biomarkers (ROS production rate, TBARS, and PC) versus all the other measured parameters' concentration data collected from HIR and IR athletes at PRE and POST session times were calculated. ROS production rate data (Figure 4$)$ were directly correlated to TBARS and PC $\left(p<0.0001, R^{2}=0.38\right.$ and $\left.R^{2}=0.40\right)$ (Figures $4(\mathrm{a})$ and $4(\mathrm{~b})$ ) and aminothiols levels (Figures 4(c)-4(l)).

TBARS concentration data were positively correlated to the following concentrations: total Cys $\left(p<0.0001, R^{2}=\right.$ $0.38)$, reduced Cys $\left(p<0.01, R^{2}=0.13\right)$, and oxidized Cys $\left(p<0.0001, R^{2}=0.38\right)$; total Hcy $\left(p<0.001, R^{2}=0.18\right)$ and reduced Hcy $\left(p<0.0001, R^{2}=0.32\right)$; total CysGly $\left(p<0.0001, R^{2}=0.36\right)$ and oxidized CysGly $(p<0.001$,
$\left.R^{2}=0.37\right) ;$ and total GSH $\left(p<0.0001, R^{2}=0.46\right)$ and oxidized GSH $\left(p<0.0001, R^{2}=0.45\right)$.

PC concentration data were positively correlated to the following concentrations: total Cys $\left(p<0.0001, R^{2}=0.30\right)$, reduced Cys $\left(p<0.05, R^{2}=0.11\right)$, and oxidized Cys $\left(p<0.0001, R^{2}=0.31\right)$; total Hcy $\left(p<0.01, R^{2}=0.18\right)$ and reduced Hcy $\left(p<0.0001, \quad R^{2}=0.27\right) ; \quad$ total CysGly $\left(p<0.0001, R^{2}=0.21\right)$ and oxidized CysGly $(p<0.001$, $\left.R^{2}=0.22\right)$; and total GSH $\left(p<0.0001, R^{2}=0.41\right)$ and oxidized GSH $\left(p<0.0001, R^{2}=0.39\right)$.

Cytokine levels increased (Table 2) and were found correlated to the increase of the correspondent ROS production, lipid peroxidation (TBARS), and protein carbonyl (PC) concentrations. IL- 6 and IL-10 were correlated with ROS 


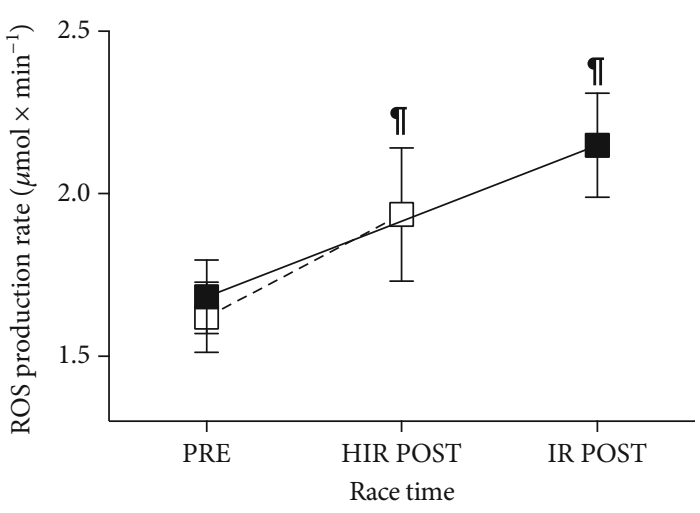

(a)

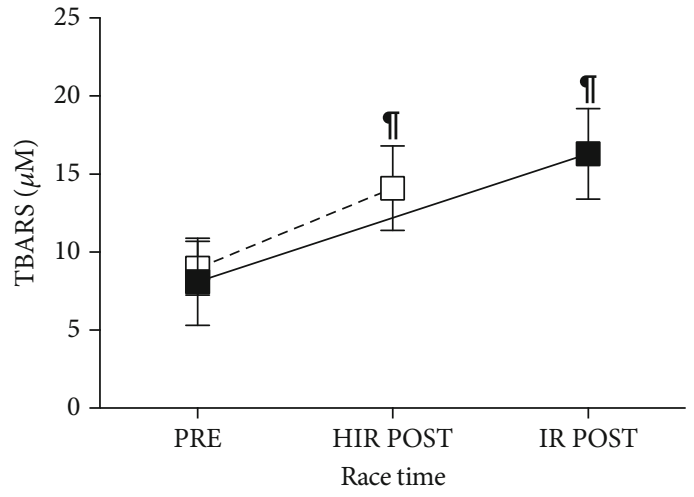

(b)

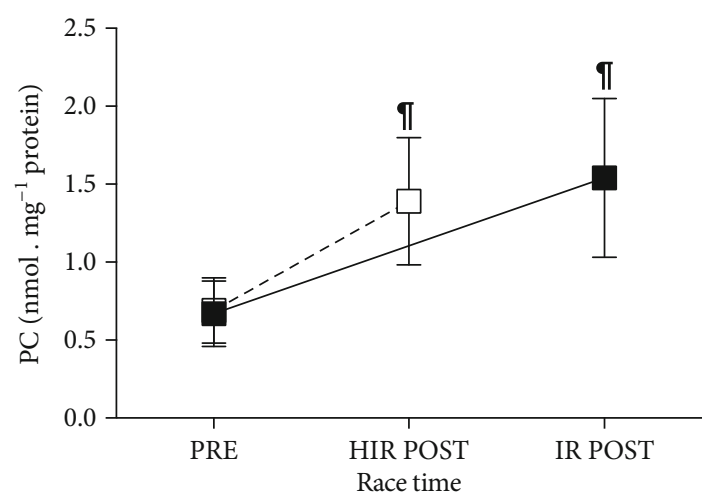

(c)

FIGURE 2: Plots of the (a) ROS production rate $\left(\mu \mathrm{mol} \cdot \mathrm{min}^{-1}\right)$; (b) TBARS $(\mu \mathrm{M})$, and (c) PC (nmol.mg ${ }^{-1}$ protein) data collected from the HIR (empty square) and IR (full square) groups. Data are expressed as the mean \pm SD. The lines connecting PRE and POST data are displayed as guide for the eyes. Changes over time (POST vs. PRE) were significant at ${ }^{\circ} p<0.0001$.

$\left(R^{2}=0.16\right.$ and $\left.R^{2}=0.16\right)$, TBARS $\left(R^{2}=0.20\right.$ and $\left.R^{2}=0.17\right)$, and PC $\left(R^{2}=0.13\right.$ and $\left.R^{2}=0.13\right)$ stating that these were not significant. Furthermore, Irisin and MCP-1 levels were found correlated with OxS biomarkers: $\operatorname{ROS}\left(R^{2}=0.21, p<0.001\right.$; $\left.R^{2}=0.31, p<0.0001\right)$, TBARS $\left(R^{2}=0.13, p<0.01 ; R^{2}=\right.$ $0.43, p<0.0001)$, and PC $\left(R^{2}=0.11, p<0.05 ; R^{2}=0.33\right.$, $p<0.0001)$.

Finally, the correlation between lymphocytes and IL-6 and IL-10 cytokine concentration data is displayed in Figures 5(a) and 5(b). In HIR and IR athletes, the levels of cytokines significantly $(p<0.01)$ increased and resulted to be directly correlated with a decrease in circulating lymphocyte concentrations.

Finally, at POST, the individual ROS production rate data was found directly correlated with the athletes' age $\left(R^{2}=0.19, \quad p<0.05\right)$, ultraendurance years of training $\left(R^{2}=0.18, p<0.05\right)$, and the days/week training activity $\left(R^{2}=0.16, p<0.05\right)$. This latter (i.e., training hours spent in two weeks before the race) was found inversely correlated with the POST IL 6 level $\left(R^{2}=-0.21, p<0.01\right)$.

\section{Discussion}

Although up to now the demands of training and competition in an ultra-endurance race are not well described, it has been suggested that triathletes do "extreme amounts of exercises" [3]. Therefore, triathlon Ironman ${ }^{\circledR}$ distance competition can be considered suitable to be studied as a very interesting model of an extreme physiological situation able to promote the deleterious actions of ROS. Most relevant novelty of the present work was the multicomprehensive set of parameters monitored and, in particular, the ROS production rate levels obtained by electron paramagnetic resonance, the only technique able to provide direct and absolute quantitative ROS levels. Oxidative damage biomarkers (TBARS and PC) were monitored too, and for the first time in a triathlon race study, aminothiols redox status in both erythrocytes and plasma was assessed. Moreover, the correlations between the reduced-oxidized forms of various aminothiols (the major nonenzymatic antioxidants in human plasma and erythrocytes), lipid and protein damages, inflammatory response, functional renal impairment, hematological parameters, and training load characteristics influence were calculated.

As widely reported in the literature, during intense aerobic exercise, the rate of oxygen consumption increases due to the significant need for ATP production and this is associated with a large oxygen flux into mitochondria of the working skeletal muscles. Enhances in oxygen consumption are strictly related to ROS production [37]. However, overwhelming ROS production may result in 
TABLE 3: Aminothiol redox status measured in plasma and red blood cells (RBCs) on HIR and IR athletes before (PRE) and immediately after (POST) the race. Concentrations of the various forms are expressed as $\mu \mathrm{mol} \cdot \mathrm{L}^{-1}$.

\begin{tabular}{|c|c|c|c|c|c|c|}
\hline \multirow{2}{*}{ Plasma } & \multicolumn{3}{|c|}{ HIR } & \multicolumn{3}{|c|}{ IR } \\
\hline & PRE & POST & $p$ value & PRE & POST & $p$ value \\
\hline \multicolumn{7}{|c|}{ Cysteine } \\
\hline Total & $239.42 \pm 17.81$ & $305.36 \pm 26.11$ & $<0.0001$ & $213.51 \pm 31.28$ & $351.38 \pm 50.19$ & 0.0007 \\
\hline Reduced & $10.08 \pm 1.01$ & $12.79 \pm 1.73$ & ns & $9.15 \pm 2.77$ & $13.92 \pm 4.04$ & ns \\
\hline Oxidized & $229.34 \pm 18.14$ & $292.57 \pm 25.99$ & $<0.0001$ & $204.35 \pm 30.04$ & $337.45 \pm 47.45$ & 0.0006 \\
\hline \multicolumn{7}{|c|}{ Homocysteine } \\
\hline Total & $6.95 \pm 0.87$ & $8.19 \pm 0.76$ & ns & $6.91 \pm 0.79$ & $8.99 \pm 1.61$ & 0.0147 \\
\hline Reduced & $0.10 \pm 0.04$ & $0.16 \pm 0.03$ & ns & $0.10 \pm 0.04$ & $0.19 \pm 0.06$ & ns \\
\hline \multicolumn{7}{|c|}{ Cysteinylglycine } \\
\hline Total & $29.66 \pm 3.06$ & $40.90 \pm 5.13$ & ns & $27.35 \pm 3.00$ & $43.24 \pm 6.25$ & 0.0004 \\
\hline Reduced & $2.34 \pm 0.32$ & $2.71 \pm 0.31$ & ns & $2.78 \pm 0.68$ & $3.17 \pm 0.75$ & ns \\
\hline Oxidized & $27.31 \pm 2.91$ & $38.19 \pm 5.18$ & ns & $24.56 \pm 2.68$ & $40.07 \pm 5.92$ & 0.0004 \\
\hline \multicolumn{7}{|c|}{ Glutathione } \\
\hline Total & $6.22 \pm 1.16$ & $8.18 \pm 1.15$ & ns & $6.47 \pm 0.54$ & $16.73 \pm 1.86$ & $<0.0001$ \\
\hline Reduced & $0.92 \pm 0.37$ & $0.92 \pm 0.39$ & ns & $0.57 \pm 0.25$ & $0.70 \pm 0.49$ & ns \\
\hline Oxidized & $5.30 \pm 1.31$ & $7.26 \pm 1.26$ & ns & $5.90 \pm 0.54$ & $16.03 \pm 1.59$ & $<0.0001$ \\
\hline \multicolumn{7}{|c|}{ RBCs } \\
\hline \multicolumn{7}{|c|}{ Cysteine } \\
\hline Total & $64.67 \pm 8.61$ & $74.69 \pm 9.23$ & ns & $62.16 \pm 7.67$ & $72.21 \pm 8.89$ & ns \\
\hline Reduced & $10.45 \pm 1.45$ & $10.85 \pm 1.53$ & ns & $11.13 \pm 1.77$ & $11.94 \pm 1.79$ & ns \\
\hline Oxidized & $54.22 \pm 8.23$ & $63.84 \pm 9.00$ & ns & $51.03 \pm 7.07$ & $60.27 \pm 8.46$ & ns \\
\hline \multicolumn{7}{|c|}{ Homocysteine } \\
\hline Total & $2.94 \pm 0.39$ & $3.11 \pm 0.43$ & ns & $2.61 \pm 0.20$ & $3.06 \pm 0.29$ & ns \\
\hline Reduced & $1.17 \pm 0.11$ & $1.09 \pm 0.13$ & ns & $1.14 \pm 0.17$ & $1.19 \pm 0.27$ & ns \\
\hline \multicolumn{7}{|c|}{ Cysteinylglycine } \\
\hline Total & $2.16 \pm 0.44$ & $2.51 \pm 0.34$ & ns & $2.17 \pm 0.41$ & $2.53 \pm 0.27$ & ns \\
\hline Reduced & $0.47 \pm 0.37$ & $0.62 \pm 0.31$ & ns & $0.56 \pm 0.17$ & $0.66 \pm 0.16$ & ns \\
\hline Oxidized & $1.69 \pm 0.73$ & $1.89 \pm 0.38$ & ns & $1.61 \pm 0.46$ & $1.87 \pm 0.35$ & ns \\
\hline \multicolumn{7}{|c|}{ Glutathione } \\
\hline Total & $1691.46 \pm 134.29$ & $1957.69 \pm 194.03$ & $<0.0001$ & $1870.35 \pm 211.98$ & $2160.42 \pm 181.61$ & $<0.0001$ \\
\hline Reduced & $1507.52 \pm 141.05$ & $1677.72 \pm 168.83$ & $<0.0001$ & $1681.73 \pm 244.14$ & $1865.54 \pm 219.23$ & $<0.0001$ \\
\hline Oxidized & $183.93 \pm 58.56$ & $279.98 \pm 71.24$ & ns & $188.62 \pm 57.34$ & $294.88 \pm 96.32$ & ns \\
\hline
\end{tabular}

oxidative modifications of lipids, proteins, and/or nucleic acids. In addition, ROS can affect apoptotic processes [38]. OxS, inflammation, and temporary renal function impairment are associated with fatigue and recovery from ultraendurance exercise.

4.1. Oxidative Stress Response. OxS represents an imbalance between oxidants, coming from ROS production, and antioxidants in favour of the former ones, leading to a redox signaling and controlling protein disruption [37].

Acute strenuous exercise has been suggested to increase $\mathrm{OxS}$, through the enhanced formation of ROS and nitrogen species [38]. According to these findings, in the present study, despite HIR and IR showing almost similar baseline
ROS production rate values, a significant ROS increase was detected at POST: HIR $+20 \%$ and IR $+28 \%$, respectively (see Figure 2(a)). Increased ROS formation after strenuous aerobic exercise includes an inadequate electron transfer through the mitochondrial respiratory chain, inflammatory responses, and heme protein autoxidation [38]. As already pointed out, overwhelming ROS production may result in oxidative lipid and protein modification, although the level of the oxidative damage, leading to alterations in redox homeostasis [39], is also directly related to the exercise type, intensity, and duration $[10,40,41]$.

Thus, different kinds of exercise seem to result in different oxidative damage levels, even if data stating the exact influence of the duration of the specific examined exercise 


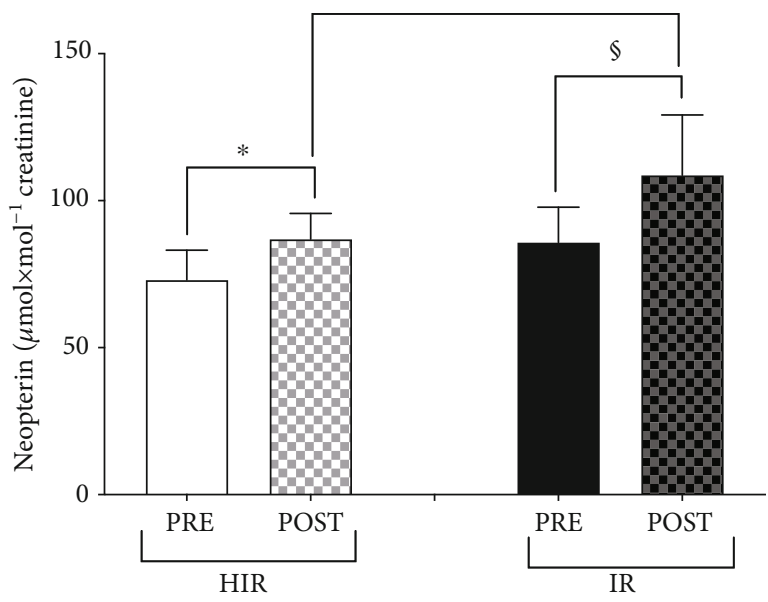

Figure 3: Neopterin concentrations $\left(\mu \mathrm{mol} \cdot \mathrm{mol}^{-1}\right.$ creatinine) measured from the HIR (empty and grey bars) and IR (full bars) athletes. Results are expressed as the mean \pm SD. Significantly different data are displayed between brackets. Changes over time (POST vs. PRE) were significant at ${ }^{*} p<0.05,{ }^{*} p<0.01$, and ${ }^{s} p<0.001$.

on the OxS level has not been ever reported before. Increased levels of various lipid peroxidation indices such as malondialdehyde (MDA), lipid hydroperoxides, $\mathrm{F}_{2}$-isoprostane measured after long-distance triathlon, or 50 and $80 \mathrm{~km}$ races have been reported in many, but not all, studies found in the literature $[8,38]$.

In the present study, the completion of an Ironman ${ }^{\circledR}$ distance triathlon (about 13- and 6-hour durations in IR and HIR groups, respectively) led to a significantly higher concentration of lipid peroxidation biomarkers (TBARS: HIR +57\%; IR +102\%) and protein carbonylation (PC: HIR +101\%; IR +126\%), when compared to PRE (Figures 2(b) and 2(c)). Indeed, a great ROS amount was generated, and consequently, they may have attacked lipids and proteins [42]. The increased lipid oxidation observed at POST may be related to the increased availability of plasma-free fatty acids, which are needed to support the long competition metabolic requests, as already reported in the literature [43].

On the other hand, protein oxidation can be initiated mainly through hydrogen abstraction from the thiol groups (to generate thiol radicals) or, possibly, from other amino acids such as methionine, as endowed by exposed susceptible functional groups [44].

Thiol groups located on various molecules are known to be central to the redox-sensitive cell signaling mechanisms by means of a reversible process representing a regulated functional switch, so playing a fundamental role in cell biology, biochemistry, and pharmacology [45]. Therefore, simultaneous determination of aminothiols may be a useful tool to study $\mathrm{OxS}$ as well as metabolic and redox regulations. The examined aminothiol redox status (see Table 3) demonstrated an increase in plasma and erythrocytes of aminothiol concentrations in both HIR and IR athletes. In particular, plasma-oxidized glutathione concentration exhibited a large increase at POST in HIR (+37\%) and IR (171\%), when compared to the other examined thiols (i.e., Cys, CysGly, and Hcy) changes.

Previously, Herrmann et al. [46] have hypothesized that exercise duration and intensity are relevant factors in Hcy modulation, while a possible reason for increased Hcy levels after endurance exercise might be the exercise-related hemoconcentration. In agreement with literature data [46], an increase of both total and reduced Hcy levels was found in the present study: HIR $+18 \%$ and $+60 \%$; IR $+30 \%$ and $+90 \%$, respectively, together with a decrease of TBW $(-4 \%)$ at POST (see Table 1). Therefore, in our opinion and taking into account that both HIR and IR groups exhibited an Hcy increase up to the $90 \%$, hemoconcentration can be regarded as a possible additional factor of influence, as suggested by Herrmann et al. [46]. This is a very important finding in the evaluation of possible detrimental effects of endurance activity, since clinical and epidemiological evidence suggests that plasmatic Hcy levels, although only moderately elevated, are associated with cardiovascular $[47,48]$ and neurodegenerative diseases $[49,50]$.

RBCs are extremely vulnerable to oxidative damage during intense extreme exercise, due to the large amount of catalytic iron, and polyunsaturated fatty acid contents that result in highly susceptible to oxidative attack [44]. In fact, the accumulation of primary products of lipid peroxidation and the formation of abnormal protein aggregates are "footprints" of RBC oxidative damage. Moreover, slightly damaged RBCs have been reported to also be susceptible targets for the attack by neutrophils.

The results of the present study showed that, even if a significant perturbation of the aminothiol redox status in plasma was demonstrated, a significant increase in total and reduced GSH was observed in $\mathrm{RBC}$. These results are in line with data previously reported in the literature, where thiol damage resulted in to some extent repair by GSH-mediated reduction [44].

4.2. Inflammation Parameters. The intense and strenuous exercise elicited an acute inflammatory response characterized by increasing in the total numbers of circulating neutrophils, monocytes (see Table 2), and OxS level, and in turn, this process stimulated a cytokine production from various cell types and unregulated the inflammatory cascade.

The response to exercise-induced muscle damage typically involves an early invasion of neutrophils that, as reported by the literature, are mobilized by cytokine-mediated demargination from the lung vasculature or released from bone marrow in response to the increased catecholamine levels $[51,52]$. Moreover, prolonged fatiguing exercise is accompanied by disturbances in total WBC (i.e., leukocytosis) and leukocyte subset counts (i.e., neutrophilia, monocytosis, and lymphopenia) [53]. Migration of leukocytes during immune surveillance and inflammation is largely determined by their response to chemokines [54].

Excessive strenuous exercise may result in enhanced enteric endotoxin translocation and a cytokine-mediated systemic inflammatory response similar to typical cytokine profile of an acute infectious episode $[55,56]$. 
TABLE 4: Correlation coefficients $\left(R^{2}\right)$ and $p$ significance levels between biochemical-hematological and OxS-selected parameters determined from all the athletes.

\begin{tabular}{|c|c|c|c|c|c|c|}
\hline \multicolumn{7}{|c|}{ Biochemical-hematological parameters } \\
\hline & ROS & $p$ value & TBARS & $p$ value & PC & $p$ value \\
\hline $\mathrm{Na}(\mathrm{mEq} / \mathrm{L})$ & $R^{2}=0.11$ & 0.0142 & - & $\mathrm{ns}$ & - & ns \\
\hline Osmolarity (mOsm/L) & $R^{2}=0.33$ & $<0.0001$ & $R^{2}=0.19$ & 0.012 & $R^{2}=0.25$ & $<0.0001$ \\
\hline Ferritin $(\mathrm{ng} / \mathrm{mL})$ & - & ns & - & ns & - & ns \\
\hline Transferrin (mg/dL) & - & ns & - & ns & - & ns \\
\hline $\mathrm{Hb}(\mathrm{g} / \mathrm{dL})$ & $R^{2}=0.11$ & 0.0132 & - & ns & - & ns \\
\hline $\operatorname{MCV}(\phi)$ & - & ns & - & ns & - & ns \\
\hline Platelets $\left(10^{9} / \mu \mathrm{L}\right)$ & - & ns & $R^{2}=0.27$ & $<0.0001$ & $R^{2}=0.17$ & 0.0021 \\
\hline Neutrophils $\left(10^{3} / \mu \mathrm{L}\right)$ & $R^{2}=0.39$ & 0.0023 & $R^{2}=0.61$ & $<0.0001$ & $R^{2}=0.52$ & $<0.0001$ \\
\hline Eosinophils $\left(10^{3} / \mu \mathrm{L}\right)$ & - & ns & - & ns & - & ns \\
\hline Lymphocyte $\left(10^{3} / \mu \mathrm{L}\right)$ & $R^{2}=0.33$ & $<0.0001$ & $R^{2}=0.50$ & $<0.0001$ & $R^{2}=0.53$ & $<0.0001$ \\
\hline Monocytes $\left(10^{3} / \mu \mathrm{L}\right)$ & - & ns & - & ns & - & ns \\
\hline HDL cholesterol (mmol/L) & $R^{2}=0.24$ & 0.0002 & $R^{2}=0.12$ & 0.0120 & - & ns \\
\hline Urea (mg/dL) & $R^{2}=0.26$ & $<0.0001$ & $R^{2}=0.36$ & $<0.0001$ & $R^{2}=0.42$ & $<0.0001$ \\
\hline Uric acid $(\mathrm{mg} / \mathrm{dL})$ & $R^{2}=0.36$ & $<0.0001$ & $R^{2}=0.24$ & 0.0002 & $R^{2}=0.28$ & $<0.0001$ \\
\hline
\end{tabular}

Our results confirmed that ultraendurance exercise leads to leukocytosis mainly due to an increase in systemic inflammation, as well as neutrophil, and monocytes levels accompanied by a relative decrease of lymphocytes [53].

In agreement with previous studies [20,57], pro- and anti-inflammatory cytokine elevations: TNF- $\alpha$, IL-1 $\beta$, IL-6, and IL-10 at POST, were observed in the present study, and the results were reported in detail by some of us [34].

Circulating levels of interleukin- (IL-) 6, IL-8, IL-1, receptor antagonist (IL-1ra), and IL-10 have been reported to increase remarkably after endurance exercises longer than $2 \mathrm{~h}$ such as marathon $[58,59]$. IL- 6 is the main cytokine present in the circulation during exercise-contracting skeletal muscle that is the main source of plasma IL-6. Its level was found to increase up to 100 -fold, depending on the intensity and duration of the endurance exercise [59].

Leukocytosis following exercise is a well-described stress/inflammatory activation phenomenon in healthy humans: our data showed that leukocytosis after triathlon competition was coincident with the increase of the inflammatory cytokines TNF- $\alpha$ and IL-6 $[55,56]$.

In agreement with literature data [60], lymphocyte count and IL-6, as well as IL-10 cytokines, resulted in being significantly correlated. Moreover, IL-6 and IL-10 levels were found to be correlated to the ROS production rate and oxidative damage of lipids and proteins. In conclusion, all these findings suggested the inflammatory cascade as a related contributor to ROS production during prolonged exercise.

4.3. Renal Functionality. Besides the biomarkers of the oxidative stress profile, an increase in systemic inflammation can also be associated to altered biochemical parameters measured in urine and in particular to then level that has previously been suggested to indicate an enhancement of the immune response [61]. Recently, high neopterin production has been associated with immune cellular activation and increased OxS [62]. Therefore, by neopterin assessment, not only the extent of cellular immune activation but also the extent of OxS can be estimated [32].

In the present study, an increase of the urinary neopterin concentration was measured immediately after the race, associated with the OxS rise. These findings suggested that "temporary" renal function impairment could also be regarded as a likely physiological response to a strenuous exercise like ultraendurance competition. Many studies showed that strenuous exercise can be associated with transitory kidney injury although whether this represents a significant insult is not clear [63-65]. Recently, a review on this topic underlined the utility to monitoring kidney function in particular in athletes with acute illness during the race (i.e., any intercurrent illness or gastrointestinal upset), exertional heat stroke, volume depletion, and use of nonsteroidal anti-inflammatory drugs that could be at a higher risk for kidney failure [66]. Further studies are needed to evaluate an optimal biomarker for kidney function assessment after intense exercise in particular in risk subjects.

4.4. Parameter Correlations. All data collected in the present study suggested that Ironman triathlon competition elucidated alterations in systemic inflammation and OxS biomarkers both in IR and in HIR. In particular, all changes indicating increased ROS production were found directly related to an increase in the oxidative stress biomarker concentration (Figures 4(a) and 4(b)) and aminothiol levels (Figures 4(c)-4(l)). These results confirm previous observations reporting analogous relationships determined under resting condition [24,67] or considering the aminothiol delta level between post- and prerunning race [8] also suggesting that the ROS amount generated during triathlon competition modulates the pathways of oxidative damage 


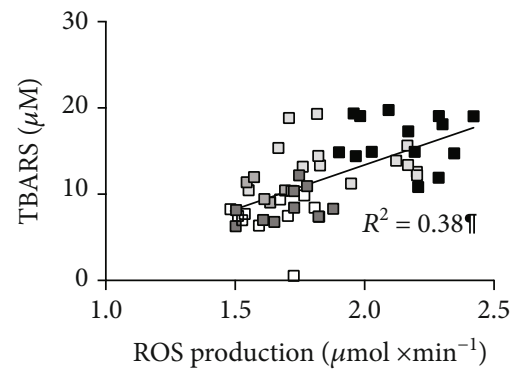

(a)

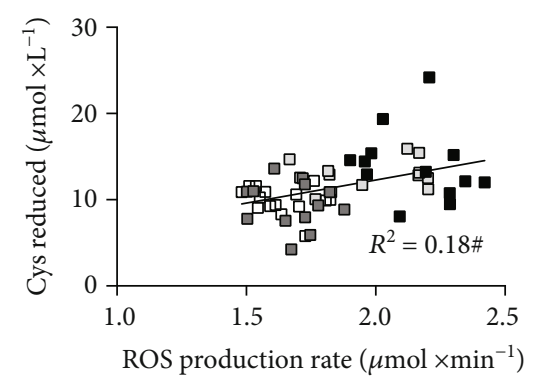

(d)

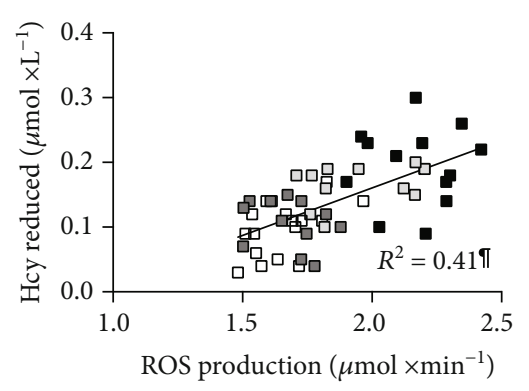

(g)

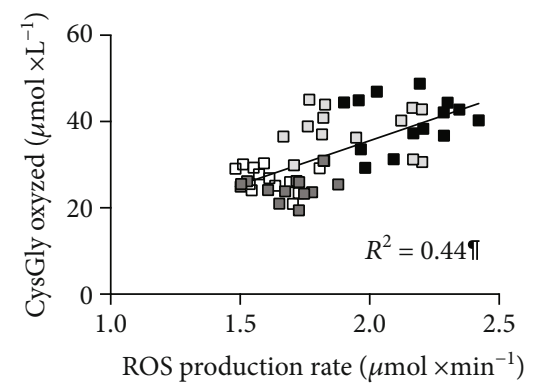

(j)

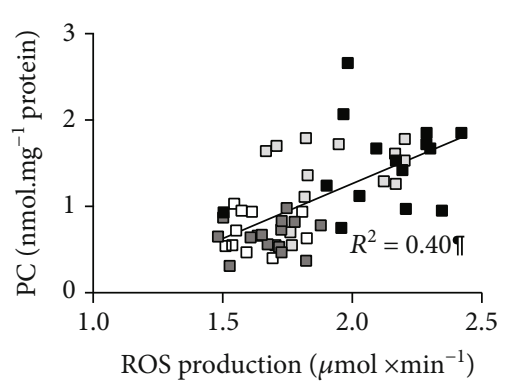

(b)

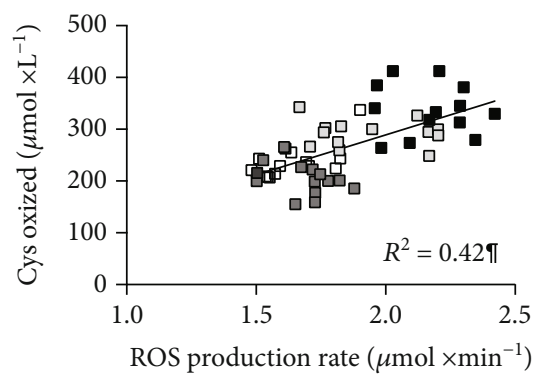

(e)

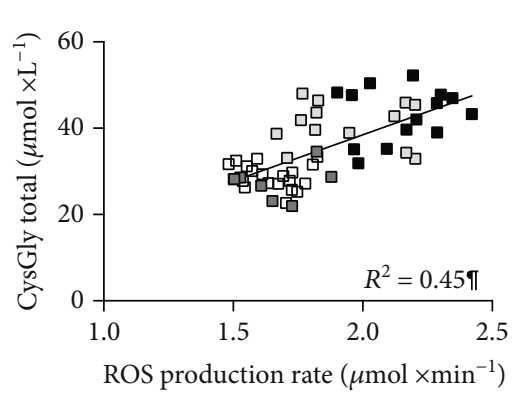

(h)

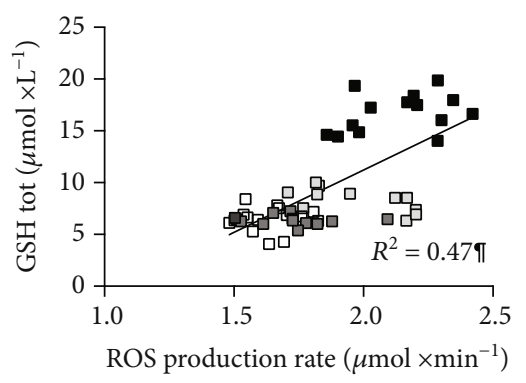

(k)

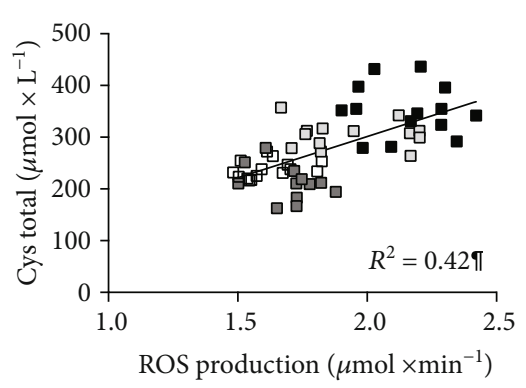

(c)

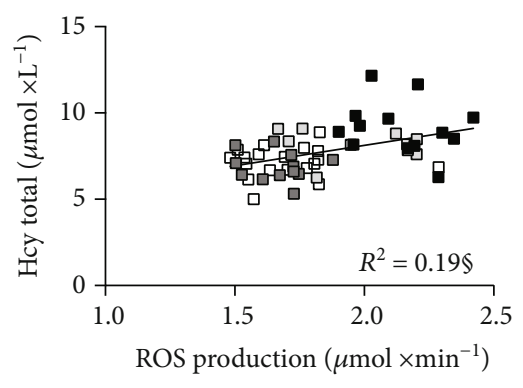

(f)

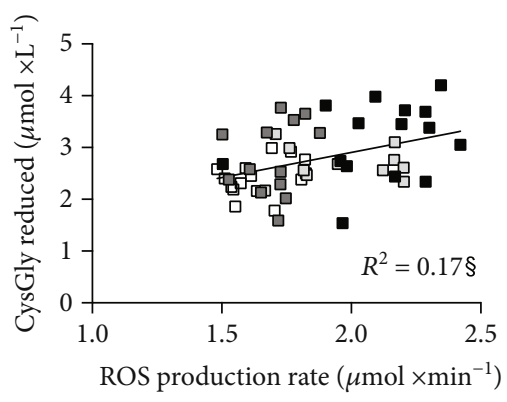

(i)

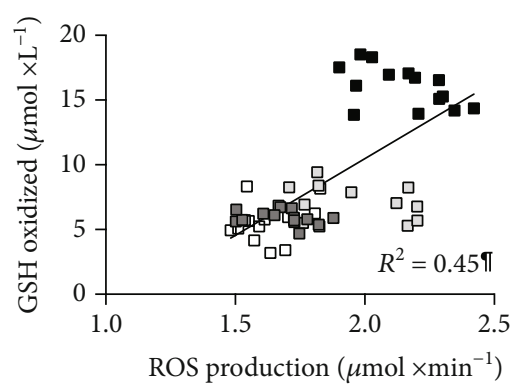

(l)

FIGURE 4: Panel plot showing the individual ROS production rate concentration values $\left(\mu \mathrm{mol} \cdot \mathrm{min}^{-1}\right)$ plotted versus the following: (a) TBARS $(\mu \mathrm{M})$; (b) PC (nmol.mg ${ }^{-1}$ protein); (c) total, (d) reduced, and (e) oxidized Cys $\left(\mu \mathrm{mol} \cdot \mathrm{L}^{-1}\right)$; (f) total and $(\mathrm{g})$ reduced Hcy $\left(\mu \mathrm{mol} \cdot \mathrm{L}^{-1}\right)$; (h) total, (i) reduced, and (j) oxidized CysGly $\left(\mu \mathrm{mol} \cdot \mathrm{L}^{-1}\right)$; $(\mathrm{k})$ total and (l) oxidized glutathione $\left(\mu \mathrm{mol} \cdot \mathrm{L}^{-1}\right)$ values obtained in HIR athletes at PRE (empty square) and POST (light grey square) and in IR athletes at PRE (dark grey square) and POST (black square). The linear regression lines (solid lines) and the correlation coefficients $\left(R^{2}\right)$ are reported in each panel. Significant relationships: ${ }^{\#} p<0.01,{ }^{\$} p<0.001$, and s $p<0.0001$.

and at the same time thiol groups are very susceptible to oxidation. Plasma aminothiols (Cys, Hcy, CysGly, and GSH) that are metabolically intercorrelated play an important role in determining the redox status of the cell environment. Thus, an altered plasma aminothiols' profile may reflect a prooxidant status of the athletes at the end of the competition.
The well-known relationship between ROS production and an individual's age [67] can surely be considered a relevant cause of the ROS production rate increase observed according to the average age of ultraendurance experience. In addition, novel findings of the present study are the observed ROS production increase consequent to the days/week training activity and the relationship between the 


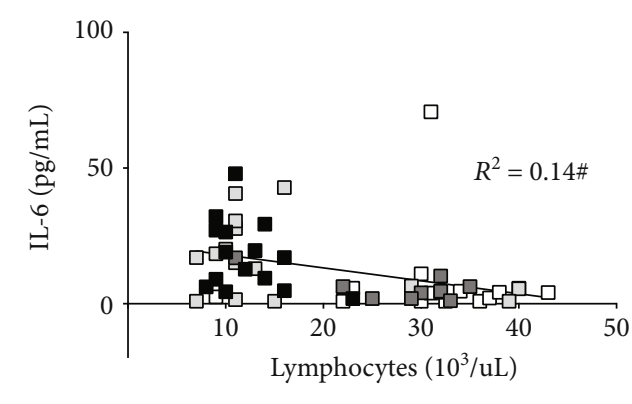

(a)

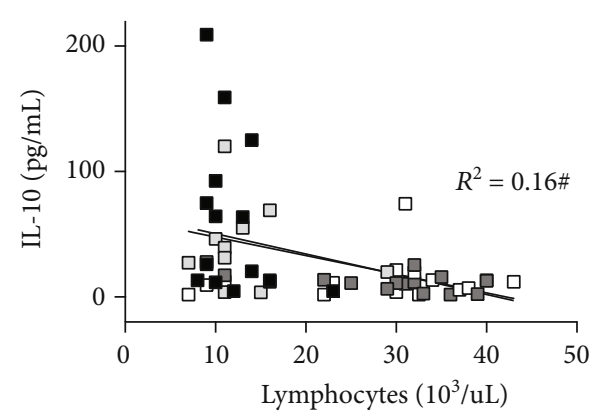

(b)

FIGURE 5: Panel plot showing the lymphocyte count $\left(10^{3} / \mu \mathrm{L}\right)$ versus cytokine (a) IL-6 and (b) IL-10 concentrations (pg/mL) obtained from HIR subjects at PRE (empty square) and POST (light grey square) and from IR subjects at PRE (dark grey square) and POST (black square). The linear regression lines (solid lines) and the correlation coefficients $\left(R^{2}\right)$ are reported in each panel. Significant relationships: ${ }^{*} p<0.01$.

preparation activity load in two weeks before race and the inflammatory marker IL-6 levels recorded from the athletes at POST. Despite the low coefficients calculated $\left(R^{2}=0.16\right.$ and 0.21 , respectively), the correlation recorded might suggest that the identification of the most appropriate training load for each subject is a crucial issue with the aim to achieve at the same time the lowest ROS production and IL-6 concentration.

\section{Limitations of the Study}

The authors are aware that the present study suffers from certain limitations. First of all, no information on postrace recovery and long-term follow up could be useful to the investigation. On the other hand, it must be pointed out that measurements carried out in the period following the race might be affected by other competitions performed by the athletes so it is actually impossible to evaluate just the single race recovery effects.

Furthermore, during the race, food intake was not standardized so the study lacked of a precise control in water and food intake.

Finally, it would have been interesting to evaluate all the parameters analyzed after each discipline: swimming, cycling, and running to understand possible different behavior in activities, but this is unreliable during a competition.

\section{Conclusions}

Altogether, the entire set of parameters monitored in the present study leads us to conclude that an intense endurance exercise, as the Ironman ${ }^{\circledR}$ distance triathlon competition, causes alterations in the oxidative stress balance (lipid and protein damage, aminothiols), highlighted by the assessed ROS overproduction, changes in inflammatory states (cytokine levels, neopterin concentration), and biochemical hematological parameters. Data indicate that a single competition of ultraendurance exercise is associated with a systemic acute and elevated $\mathrm{OxS}$ response with a disturbance in the oxidant/antioxidant balance.
Further studies are needed to shed light about the volume and intensity of exercise which can be considered "healthy" and to investigate possible long-term consequences of repeated and heavy stress as those supported by the triathletes. At the same time, it would be helpful that other biomarkers suitable to increase the specificity between true pathology and physiological adaptation would be identified and to clarify the clinical relevance of such acute changes.

We are all aware that, as recently reported, the endurance and ultraendurance activity is probably the most popular sport worldwide, and it is practiced for recreational, health, and competitive purposes [68]; nonetheless, altogether, the data obtained in the present study put in evidence that an appropriate training time is essential to achieve at the same time the lowest ROS production and IL- 6 concentration; in other words, the "right" physical activity will have beneficial effects.

$\begin{array}{ll}\text { Abbreviations } & \\ \text { IR: } & \text { Ironman }{ }^{\circledR} \\ \text { HIR: } & \text { Half Ironman }{ }^{\circledR} \\ \text { BMI: } & \text { Body mass index } \\ \text { SaO }: & \text { Arterial oxygen saturation } \\ \text { HR: } & \text { Heart rate } \\ \text { FM: } & \text { Fat mass } \\ \text { FFM: } & \text { Fat-free mass } \\ \text { TBW: } & \text { Total body water } \\ \text { SBP: } & \text { Systolic blood pressure } \\ \text { DBP: } & \text { Diastolic blood pressure } \\ \text { OxS: } & \text { Oxidative stress } \\ \text { EPR: } & \text { Electron paramagnetic resonance } \\ \text { ROS: } & \text { Reactive oxygen species } \\ \text { TBARS: } & \text { Thiobarbituric acid reactive substances } \\ \text { PC: } & \text { Protein carbonyls } \\ \text { GSH: } & \text { Glutathione } \\ \text { Hcy: } & \text { Homocysteine } \\ \text { Cys: } & \text { Cysteine } \\ \text { CysGly: } & \text { Cysteinylglycine } \\ \text { TNF- } \alpha: & \text { Tumor necrosis factor- } \alpha \\ \text { IL-1 } \beta, \text { IL-6, IL-10: } & \text { Interleukin-1 } \beta, 6, \text { and 10 } \\ \text { MCP-1: } & \text { Monocyte Chemoattractant Protein-1. } \\ & \end{array}$




\section{Data Availability}

The data used to support the findings of this study are included within the article.

\section{Conflicts of Interest}

The authors declare that there are no conflicts of interest.

\section{Authors' Contributions}

SMS conceived and designed the research; analyzed the data and statistical analysis; prepared the figures; drafted, edited, and revised manuscript; and approved the final version of the manuscript submitted. MG is assigned to the statistical analysis; drafted, edited, and revised the manuscript; and approved the final version of manuscript submitted. AV performed the experiments and critically revised the intellectual content of the manuscript and approved the final version of manuscript submitted. DN analyzed the data and approved the final version of manuscript submitted. MC performed the experiments and approved the final version of the manuscript submitted. GG was involved in the study design and final approval of the manuscript submitted. RB performed the experiments and approved the final version of the manuscript submitted. MM performed the experiments, analyzed the data, and approved the final version of the manuscript submitted. AC, SM, and FG analyzed the data and approved the final version of the manuscript submitted. LP conceived and designed the research, interpreted the data, drafted and wrote the manuscript, and approved the final version of the paper.

\section{Acknowledgments}

The authors wish to thank the Scientific Commission of Italian Federation of Sport Medicine for the financial support. They are grateful to all the athletes that participated voluntarily in the experimentation and the organizing committee of the Elbaman ${ }^{\circledR}$.

\section{References}

[1] W. E. Garrett and D. T. Kirkendall, Exercise and sport science, Lippincott Williams \& Wilkins, 2000.

[2] N. Etxebarria, I. Mujika, and D. B. Pyne, "Training and competition readiness in triathlon," Sports, vol. 7, no. 5, p. 101, 2019.

[3] V. Vleck, G. P. Millet, and F. B. Alves, "The impact of triathlon training and racing on athletes' general health," Sports Medicine, vol. 44, no. 12, pp. 1659-1692, 2014.

[4] L. L. Smith, A. Anwar, M. Fragen, C. Rananto, R. Johnson, and D. Holbert, "Cytokines and cell adhesion molecules associated with high-intensity eccentric exercise," European Journal of Applied Physiology, vol. 82, no. 1-2, pp. 61-67, 2000.

[5] T. Hew-Butler, M. Collins, A. Bosch et al., "Maintenance of plasma volume and serum sodium concentration despite body weight loss in Ironman triathletes," Clinical Journal of Sport Medicine, vol. 17, no. 2, pp. 116-122, 2007.

[6] K. Suzuki, J. Peake, K. Nosaka et al., "Changes in markers of muscle damage, inflammation and HSP70 after an Ironman
Triathlon race," European Journal of Applied Physiology, vol. 98, no. 6, pp. 525-534, 2006.

[7] S. Mrakic-Sposta, M. Gussoni, M. Montorsi, S. Porcelli, and A. Vezzoli, "Assessment of a standardized ROS production profile in humans by electron paramagnetic resonance," Oxidative Medicine and Cellular Longevity, vol. 2012, Article ID 973927, 10 pages, 2012.

[8] A. Vezzoli, C. Dellanoce, S. Mrakic-Sposta et al., "Oxidative stress assessment in response to ultraendurance exercise: thiols redox status and ROS production according to duration of a competitive race," Oxidative Medicine and Cellular Longevity, vol. 2016, Article ID 6439037, 13 pages, 2016.

[9] W. L. Knez, D. G. Jenkins, and J. S. Coombes, "Oxidative stress in half and full Ironman triathletes," Medicine \& Science in Sports \& Exercise, vol. 39, no. 2, pp. 283-288, 2007.

[10] R. A. Pinho, L. A. Silva, C. A. Pinho et al., "Oxidative stress and Inflammatory parameters after an ironman race," Clinical Journal of Sport Medicine, vol. 20, no. 4, pp. 306-311, 2010.

[11] G. J. Rietjens, H. Kuipers, F. Hartgens, and H. A. Keizer, "Red blood cell profile of elite olympic distance triathletes. A threeyear follow-up," International Journal of Sports Medicine, vol. 23, no. 6, pp. 391-396, 2002.

[12] J. E. Turner, S. J. Bennett, J. A. Bosch, H. R. Griffiths, and S. Aldred, "Ultra-endurance exercise: unanswered questions in redox biology and immunology," Biochemical Society Transactions, vol. 42, no. 4, pp. 989-995, 2014.

[13] K. Ujka, L. Bastiani, G. D'Angelo et al., "Enhanced rightchamber remodeling in endurance ultra-trail athletes compared to marathon runners detected by standard and speckle-tracking echocardiography," Frontiers in Physiology, vol. 8, p. 527, 2017.

[14] D. M. Bailey, I. S. Young, J. McEneny et al., "Regulation of free radical outflow from an isolated muscle bed in exercising humans," American Journal of Physiology Heart and Circulatory Physiology, vol. 287, no. 4, pp. H1689-H1699, 2004.

[15] J. E. Turner, N. J. Hodges, J. A. Bosch, and S. Aldred, "Prolonged depletion of antioxidant capacity after ultraendurance exercise," Medicine and Science in Sports and Exercise, vol. 43, no. 9, pp. 1770-1776, 2011.

[16] M. G. Nikolaidis, A. Kyparos, C. Spanou, V. Paschalis, A. A. Theodorou, and I. S. Vrabas, "Redox biology of exercise: an integrative and comparative consideration of some overlooked issues," Journal of Experimental Biology, vol. 215, no. 10, pp. 1615-1625, 2012.

[17] R. Kohen and A. Nyska, "Oxidation of biological systems: oxidative stress phenomena, antioxidants, redox reactions, and methods for their quantification," Toxicologic Pathology, vol. 30, no. 6, pp. 620-650, 2002.

[18] Y. McLeay, S. Stannard, S. Houltham, and C. Starck, "Dietary thiols in exercise: oxidative stress defence, exercise performance, and adaptation," Journal of the International Society of Sports Nutrition, vol. 14, no. 1, 2017.

[19] E. T. C. Collins, A. M. James, K. E. Menger, H. M. Cocheme, and M. P. Murphy, "Mitochondrial redox signalling at a glance," Journal of Cell Science, vol. 125, no. 7, p. 1837, 2012.

[20] C. Vassalle, P. Piaggi, N. Weltman et al., "Innovative approach to interpret the variability of biomarkers after ultra-endurance exercise: the multifactorial analysis," Biomarkers in Medicine, vol. 8, no. 6, pp. 881-891, 2014.

[21] D. M. Bailey, L. Lawrenson, J. McEneny et al., "Electron paramagnetic spectroscopic evidence of exercise-induced free 
radical accumulation in human skeletal muscle," Free Radical Research, vol. 41, no. 2, pp. 182-190, 2007.

[22] M. Valko, D. Leibfritz, J. Moncol, M. T. Cronin, M. Mazur, and J. Telser, "Free radicals and antioxidants in normal physiological functions and human disease," The International Journal of Biochemistry \& Cell Biology, vol. 39, no. 1, pp. 44-84, 2007.

[23] S. Suzen, H. Gurer-Orhan, and L. Saso, "Detection of reactive oxygen and nitrogen species by electron paramagnetic resonance (EPR) technique," Molecules, vol. 22, no. 1, p. 181, 2017.

[24] K. K. Griendling, R. M. Touyz, J. L. Zweier et al., "Measurement of reactive oxygen species, reactive nitrogen species, and redox-dependent signaling in the cardiovascular system: a scientific statement from the American Heart Association," Circulation Research, vol. 119, no. 5, pp. e39-e75, 2016.

[25] S. Mrakic-Sposta, M. Gussoni, S. Moretti et al., "Effects of mountain ultra-marathon running on ROS production and oxidative damage by micro-invasive analytic techniques," PLoS One, vol. 10, no. 11, p. e0141780, 2015.

[26] M. E. McMenamin, J. Himmelfarb, and T. D. Nolin, "Simultaneous analysis of multiple aminothiols in human plasma by high performance liquid chromatography with fluorescence detection," Journal of Chromatography B, vol. 877, no. 28, pp. 3274-3281, 2009.

[27] F. Q. Schafer and G. R. Buettner, "Redox environment of the cell as viewed through the redox state of the glutathione disulfide/glutathione couple," Free Radical Biology and Medicine, vol. 30, no. 11, pp. 1191-1212, 2001.

[28] K. Ostrowski, P. Schjerling, and B. K. Pedersen, "Physical activity and plasma interleukin-6 in humans-effect of intensity of exercise," European Journal of Applied Physiology, vol. 83, no. 6, pp. 512-515, 2000.

[29] B. K. Pedersen and L. Hoffman-Goetz, "Exercise and the immune system: regulation, integration, and adaptation," Physiological Reviews, vol. 80, no. 3, pp. 1055-1081, 2000.

[30] R. A. Pinho, M. E. Andrades, M. R. Oliveira et al., "Imbalance in SOD/CAT activities in rat skeletal muscles submitted to treadmill training exercise," Cell Biology International, vol. 30, no. 10, pp. 848-853, 2006.

[31] J. G. Cannon and J. B. BlumbergO. Ha"nninen, "Acute phase immune responses in exercise," in Handbook of Oxidants and Antioxidants in Exercise, C. K. Sen and L. Packer, Eds., pp. 177-194, Elsevier, Amsterdam, the Netherlands, 2000.

[32] C. Murr, B. Widner, B. Wirleitner, and D. Fuchs, "Neopterin as a marker for immune system activation," Current Drug Metabolism, vol. 3, no. 2, pp. 175-187, 2002.

[33] P. Zis, A. Strydom, D. Buckley, D. Adekitan, and P. C. McHugh, "Cognitive ability in Down syndrome and its relationship to urinary neopterin, a marker of activated cellular immunity," Neuroscience Letters, vol. 636, pp. 254-257, 2017.

[34] M. Comassi, E. Vitolo, L. Pratali et al., "Acute effects of different degrees of ultra-endurance exercise on systemic inflammatory responses," Internal Medicine Journal, vol. 45, no. 1, pp. 74-79, 2015.

[35] S. Mrakic-Sposta, M. Gussoni, S. Porcelli et al., "Training Effects on ROS Production Determined by Electron Paramagnetic Resonance in Master Swimmers," Oxidative Medicine and Cellular Longevity, vol. 2015, Article ID 804794, 8 pages, 2015.

[36] C. Dellanoce, L. Cozzi, S. Zuddas, L. Pratali, and R. Accinni, "Determination of different forms of aminothiols in red blood cells without washing erythrocytes," Biomedical Chromatography, vol. 28, no. 3, pp. 327-331, 2014.

[37] B. Halliwelland and J. M. C. Gutteridge, Free Radicals in Biology and Medicine, Clarendon Press, New York, NY, USA, 4th edition edition, 2007.

[38] K. H. Wagner, S. Reichhold, C. Hölzl et al., "Well-trained, healthy triathletes experience no adverse health risks regarding oxidative stress and DNA damage by participating in an ultraendurance event," Toxicology, vol. 278, no. 2, pp. 211-216, 2010.

[39] N. A. Lewis, C. Towey, G. Bruinvels, G. Howatson, and C. R. Pedlar, "Effects of exercise on alterations in redox homeostasis in elite male and female endurance athletes using a clinical point-of-care test," Applied Physiology, Nutrition, and Metabolism, vol. 41, no. 10, pp. 1026-1032, 2016.

[40] M. Lamprecht, J. F. Greilberger, G. Schwaberger, P. Hofmann, and K. Oettl, "Single bouts of exercise affect albumin redox state and carbonyl groups on plasma protein of trained men in a workload-dependent manner," Journal of Applied Physiology, vol. 104, no. 6, pp. 1611-1617, 2008.

[41] J. S. Wang, T. Lee, and S. E. Chow, "Role of exercise intensities in oxidized low-density lipoprotein-mediated redox status of monocyte in men," Journal of Applied Physiology, vol. 101, no. 3, pp. 740-744, 2006.

[42] B. Poljsak, D. Šuput, and I. Milisav, "Achieving the balance between ROS and antioxidants: when to use the synthetic antioxidants," Oxidative Medicine and Cellular Longevity, vol. 2013, Article ID 956792, 11 pages, 2013.

[43] M. Fernström, L. Bakkman, M. Tonkonogi et al., "Reduced efficiency, but increased fat oxidation, in mitochondria from human skeletal muscle after 24-h ultraendurance exercise," Journal of Applied Physiology, vol. 102, no. 5, pp. 1844-1849, 2007.

[44] J. A. Smith, "Exercise, training and red blood cell turnover," Sports Medicine, vol. 19, no. 1, pp. 9-31, 1995.

[45] E. C. Gomes, A. N. Silva, and M. R. de Oliveira, "Oxidants, antioxidants, and the beneficial roles of exercise-induced production of reactive species," Oxidative Medicine and Cellular Longevity, vol. 2012, Article ID 756132, 12 pages, 2012.

[46] M. Herrmann, H. Schorr, R. Obeid et al., "Homocysteine increases during endurance exercise," Clinical Chemistry and Laboratory Medicine, vol. 41, no. 11, pp. 1518-1524, 2003.

[47] P. J. Barter and K. A. Rye, "Homocysteine and cardiovascular disease. Is HDL the link?," Circulation Research, vol. 99, no. 6, pp. 565-566, 2006.

[48] P. Ganguly and S. F. Alam, "Role of homocysteine in the development of cardiovascular disease," Nutrition Journal, vol. 14, no. 1, pp. 1-10, 2015.

[49] D. P. Jones, J. L. Carlson, V. C. Mody Jr., J. Cai, M. J. Lynn, and P. Sternberg Jr., "Redox state of glutathione in human plasma," Free Radical Biology and Medicine, vol. 28, no. 4, pp. 625-635, 2000.

[50] M. Sharma, M. Tiwari, and R. K. Tiwari, "Hyperhomocysteinemia: impact on neurodegenerative diseases," Basic \& Clinical Pharmacology \& Toxicology, vol. 117, no. 5, pp. 287-296, 2015.

[51] J. G. Tidball, "Inflammatory processes in muscle injury and repair," American Journal of Physiology. Regulatory, Integrative and Comparative Physiology, vol. 288, no. 2, pp. R345R353, 2005.

[52] T. A. Butterfield, T. M. Best, and M. A. Merrick, "The dual roles of neutrophils and macrophages in inflammation: a 
critical balance between tissue damage and repair," Journal of Athletic Training, vol. 41, no. 4, pp. 457-465, 2006.

[53] Y. O. Shin and J. B. Lee, "Leukocyte chemotactic cytokine and leukocyte subset responses during ultra-marathon running," Cytokine, vol. 61, no. 2, pp. 364-369, 2013.

[54] S. Paoletti, V. Petkovic, S. Sebastiani, M. G. Danelon, M. Uguccioni, and B. O. Gerber, "A rich chemokine environment strongly enhances leukocyte migration and activities," Blood, vol. 105, no. 9, pp. 3405-3412, 2005.

[55] S. K. Gill, A. Teixeira, L. Rama et al., "Circulatory endotoxin concentration and cytokine profile in response to exertionalheat stress during a multi-stage ultra-marathon competition," Exercise Immunology Review, vol. 21, pp. 114-128, 2015.

[56] S. K. Gill, J. Hankey, A. Wright et al., "The impact of a 24-h ultra-marathon on circulatory endotoxin and cytokine profile," International Journal of Sports Medicine, vol. 36, no. 8, pp. 688-695, 2015.

[57] C. McLachlan and P. Mossop, "Are elevations of N-terminal probrain natriuretic peptide in endurance athletes after prolonged strenuous exercise due to systemic inflammatory cytokines?" American Heart Journal, vol. 152, no. 1, p. e1, 2006.

[58] M. A. Febbraio and B. K. Pedersen, "Muscle-derived interleukin-6: mechanisms for activation and possible biological roles," The FASEB Journal, vol. 16, no. 11, pp. 1335-1347, 2002.

[59] K. Sugama, K. Suzuki, K. Yoshitani, K. Shiraishi, and T. Kometani, "Urinary excretion of cytokines versus their plasma levels after endurance exercise," Exercise Immunology Review, vol. 19, pp. 29-48, 2013.

[60] K. Sugama, K. Suzuki, K. Yoshitani et al., "Changes of thioredoxin, oxidative stress markers, inflammation and muscle/renal damage following intensive endurance exercise," Exercise Immunology Review, vol. 21, pp. 130-142, 2015.

[61] C. Huber, J. R. Batchelor, D. Fuchs et al., "Immune responseassociated production of neopterin. Release from macrophages primarily under control of interferon-gamma," The Journal of Experimental Medicine, vol. 160, no. 1, pp. 310-316, 1984.

[62] Z. Shao, R. Zhang, K. Shrestha et al., "Usefulness of elevated urine neopterin levels in assessing cardiac dysfunction and exercise ventilation inefficiency in patients with chronic systolic heart failure," The American Journal of Cardiology, vol. 113, no. 11, pp. 1839-1843, 2014.

[63] K. J. Kosunen and A. J. Pakarinen, "Plasma renin, angiotensin II, and plasma and urinary aldosterone in running exercise," Journal of Applied Physiology, vol. 41, no. 1, pp. 26-29, 1976.

[64] R.-M. McAllister, "Adaptations in control of blood flow with training: splanchnic and renal blood flows," Medicine and Science in Sports and Exercise, vol. 30, no. 3, pp. 375-381, 1998.

[65] L. Röcker, K. A. Kirsch, B. Heyduck, and H. U. Altenkirch, "Influence of prolonged physical exercise on plasma volume, plasma proteins, electrolytes, and fluid-regulating hormones," International Journal of Sports Medicine, vol. 10, no. 4, pp. 270-274, 1989.

[66] L. E. Hodgson, E. Walter, R. M. Venn et al., “Acute kidney injury associated with endurance events-is it a cause for concern? A systematic review," BMJ Open Sport \& Exercise Medicine, vol. 3, no. 1, article e000093, 2017.
[67] S. Mrakic-Sposta, M. Gussoni, M. Montorsi, S. Porcelli, and A. Vezzoli, "A quantitative method to monitor reactive oxygen species production by electron paramagnetic resonance in physiological and pathological conditions," Oxidative Medicine and Cellular Longevity, vol. 2014, Article ID 306179, 10 pages, 2014.

[68] E. S. Barros, D. C. Nascimento, J. Prestes et al., “Acute and chronic effects of endurance running on inflammatory markers: a systematic review," Frontiers Physiology, vol. 8, p. 779, 2017. 


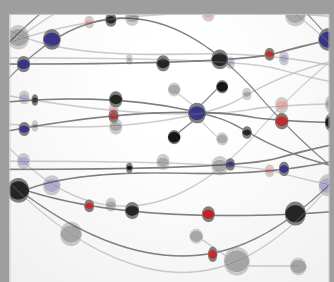

The Scientific World Journal
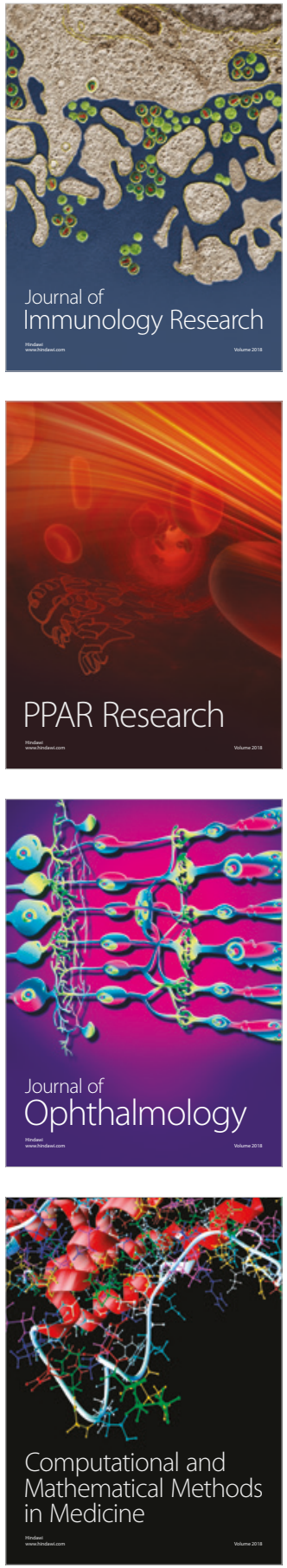

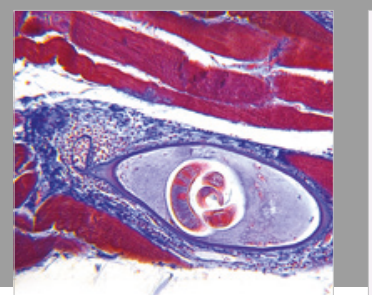

Gastroenterology Research and Practice

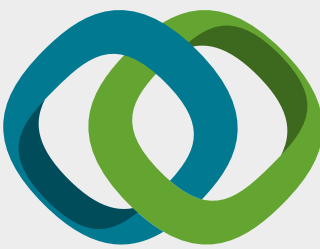

\section{Hindawi}

Submit your manuscripts at

www.hindawi.com
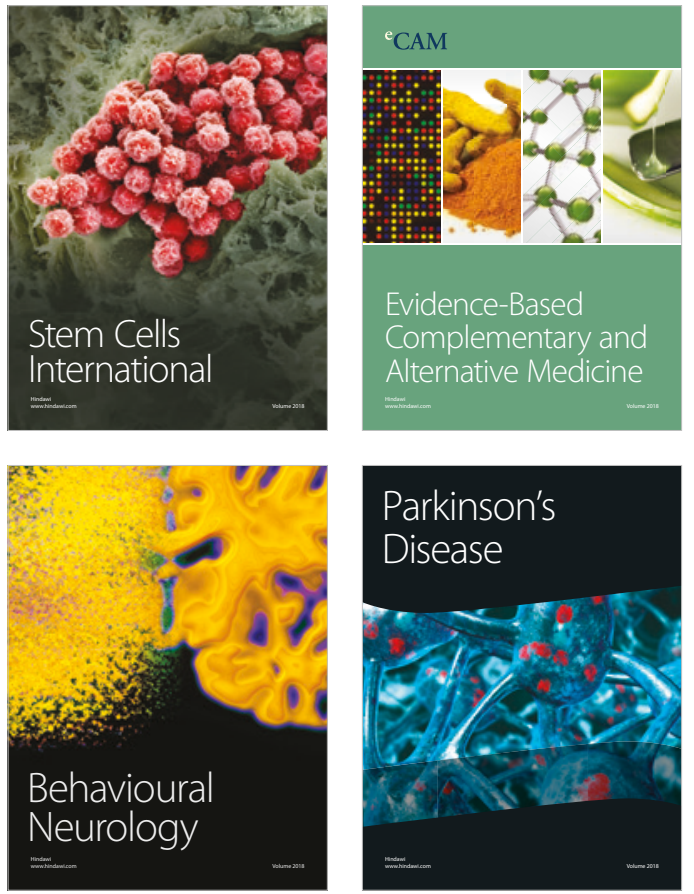

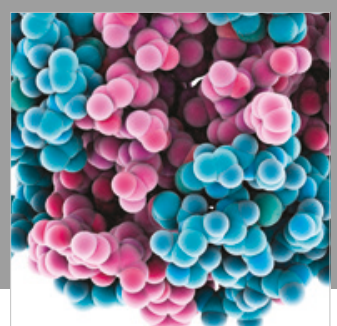

ournal of

Diabetes Research

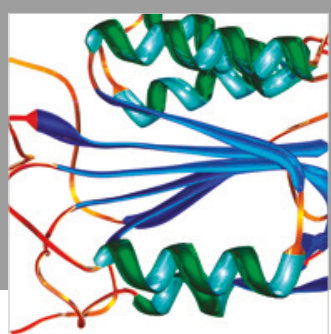

Disease Markers
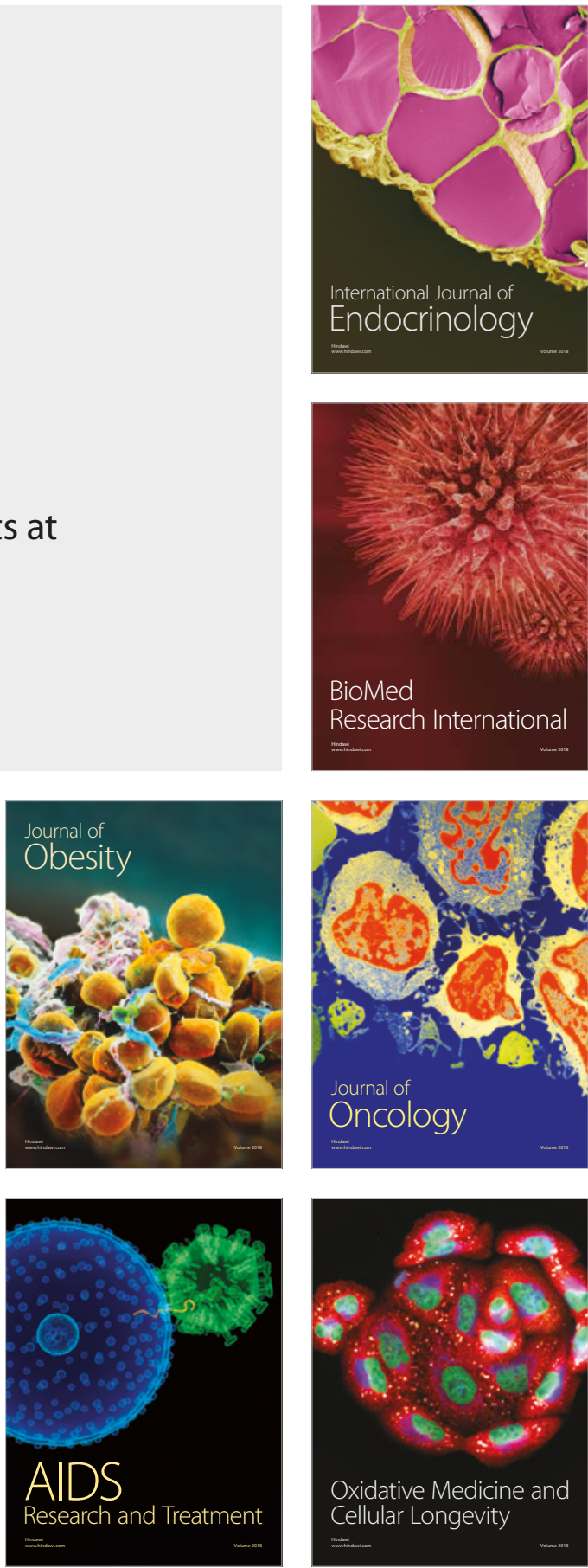